\title{
Wahl von DMV-Präsidiumsmitgliedern ab 2018
}

\author{
Vorstellung der Kandidatinnen und Kandidaten
}

Der Präsident der DMV ruft alle DMV-Mitglieder auf, sich an der Wahl von Präsidiumsmitgliedern für die Amtszeit ab dem 1.1.2018 zu beteiligen. Bei der Wahl sind drei Präsidiumsplätze zu besetzen. Es bewerben sich vier

\section{Ilka Agricola}

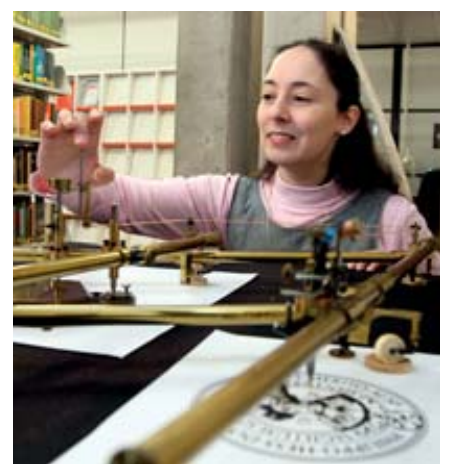

Geboren 1973 in Den Haag, aufgewachsen in München. Studium der Physik mit Nebenfach Mathematik an der TU und LMU München (Diplom 1996). Danach Gastwissenschaftlerin an der Rutgers University in New Jersey bei Roe Goodman, ab November 1997 wissenschaftli-

che Mitarbeiterin an der Humboldt-Universität zu Berlin bei Thomas Friedrich. Promotion im Jahr 2000 über Die Frobenius-Zerlegung auf algebraischen G-Mannigfaltigkeiten, die auch mit dem Humboldt-Preis ausgezeichnet wurde. Im WS 2001/o2 beurlaubt zu Gastaufenthalten an die Max-Planck-Institute in Leipzig und Potsdam. Von 2003 bis 2008 Leiterin einer von der VolkswagenStiftung finanzierten Nachwuchsgruppe an der HU Berlin, 2004 Habilitation in Mathematik an der Universität Greifswald. 1997 bis 2004 Mitglied im SFB 288 Differentialgeometrie und Quantenphysik. Mitautorin der Lehrbücher Elementargeometrie (1. Auflage 2005) und Vektoranalysis (1. Auflage 2001), beide erschienen im Vieweg-Verlag und in englischer Übersetzung bei der AMS.

2008 Rufe auf eine Professur an der Katholischen Universität Leuven (Belgien) sowie auf $\mathrm{W}_{3}$-Professuren an den Universitäten Marburg und Bremen, ab Oktober 2008 W3-Professorin für Mathematik an der PhilippsUniversität Marburg und Leiterin der Mathematischen Modellsammlung. Längere Gastaufenthalte in Wien und Seoul. 2014 Ruf an die Universität Stuttgart (abgelehnt). Seit 2014 Dekanin des Fachbereichs Mathematik und Informatik. Seit 2015 Editor in Chief der Zeitschrift Annals of Global Analysis and Geometry (Springer-Verlag) gemeinsam mit Frank Duzaar (Erlangen); Mitglied im Editorial Board der Communications in Mathematik und der Mathematischen Semesterberichte.

(Mit-)Organisatorin diverser Fachtagungen und Sommerschulen, etwa Special Geometries in Mathematical Phy-
Kandidatinnen und Kandidaten. Diese stellen sich hier vor. Die Wahlunterlagen (Stimmzettel, zwei Umschläge) liegen diesem Heft bei. sics (Kühlungsborn, 2006 und 2008), Seminar Sophus Lie (2001, 2009, 2011, 2014), Spring School The Weil representation and its applications (Marburg, 2013), Meeting of the German Chapter of European Women in Mathematics (Rauischholzhausen, 2015), Workshop on Almost Hermitian and Contact Geometry (Bedlewo/PL, 2015), 1. Marburger Arbeitsgemeinschaft Mathematik on the Hopf problem (Marburg, 2017). 2016 Preisträgerin des Ars LegendiFakultätenpreises für Mathematik und Naturwissenschaften in der Kategorie Mathematik (siehe Mitteilungen 24-4 (2016), S. 226-229).

In der Forschung beschäftige ich mich mit Fragen der Differentialgeometrie und der globalen Analysis, genauer: mit speziellen Geometrien auf Mannigfaltigkeiten (fastkomplexe Strukturen, Kontakt-Strukturen, $G_{2}$-Strukturen, usw.), Holonomie-Theorie von Zusammenhängen, SpinGeometrie und Dirac-Operatoren. Dabei fasziniert mich immer wieder, wie subtil die geometrischen Eigenschaften eines Raumes, etwa seine Krümmung, mit seinen Symmetrien (unter einer Lie-Gruppe G) und der Lösbarkeit gewisser überdeterminierter Differentialgleichungen auf diesem Raum zusammenhängen.

Zudem schätze ich einen nichtfachlichen Aspekt meiner Arbeit besonders: Wissenschaft leistet einen erheblichen Beitrag zur Völkerverständigung und ich bin dankbar, im Austausch mit Kollegen andere Länder, Kulturen und Meinungen kennenlernen zu können und so meinen persönlichen Horizont stetig zu erweitern.

Das Bild der Mathematik in der öffentlichen Wahrnehmung ist nicht sehr kenntnisreich und selten ihrem Ansehen förderlich. Die DMV ist der richtige Ort, um diesen Eindruck zu korrigieren, die Bedeutung mathematischer Grundlagenforschung und der Mathematik als High-TechWissenschaft zu vermitteln und die gesellschaftliche Relevanz der Mathematik als Strukturwissenschaft für alle - auch für Schüler! - hervorzuheben. Gleichzeitig kann nur eine starke Gemeinschaft die Belange der Mathematiker kommunizieren, etwa im Publikationswesen oder bei der Forschungsförderung, und ein Forum bieten für Vernetzung, Austausch und Meinungsbildung der ganzen community. Hierfür möchte ich mich sehr gerne einsetzen und kandidiere deswegen für einen der frei werdenden Plätze im Präsidium der DMV. 


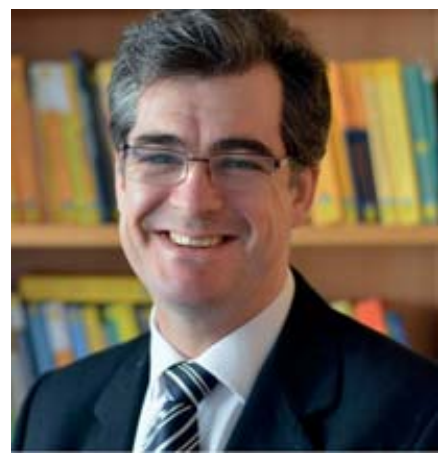

Geboren 1972, verheiratet mit Elena Kaßmann (Musikerin), vier Kinder. Studium der Mathematik und der Volkswirtschaftslehre bis zum Vordiplom an der Universität Konstanz, danach ein Jahr in Sankt Petersburg und schließlich bis zum Diplom (Mathematik) an der Rheinischen Friedrich-Wilhelms-Universität Bonn. 2001 Promotion in Bonn, 2002-2004 Postdoc mit vollem Lehrdeputat an der University of Connecticut, 2004-2007 Assistent von Prof. Dr. Frehse am Institut für Angewandte Mathematik in Bonn, 2007 Habilitation. 2008-2009 Assistent des Vorstands der Allianz Lebensversicherungs-AG, seit dem Sommersemester 2010 Professor für Mathematik an der Universität Bielefeld. Stipendien: Studienstiftung (Studium), DAAD (Auslandsjahr), BASF (Studieninitiative), DFG (Heisenberg Stipendium). Ich bin Gründungsmitglied und Sprecher des 2016 eingerichteten Internationalen Graduiertenkollegs zwischen der Universität Bielefeld und der Seoul National University. Die Betreuung von Doktorandinnen und Doktoranden aus drei Kontinenten macht mir viel Freude. Im kürzlich bewilligten Sonderforschungsbereich 1283 leite ich ein Teilprojekt. Gegenwärtig bin ich Vorsitzender des Senats der Universität Bielefeld.

Mein Schwerpunkt in der Forschung liegt in den Bereichen Partielle Differentialgleichungen und Stochastische Analysis. Insbesondere interessiere ich mich zur Zeit für die Analysis nichtlokaler Operatoren und der zugehörigen Sprungprozesse. Mein Ziel ist es, Mathematik weiterzuentwickeln, zum einen als eigenständige Wissenschaft, zum anderen als Grundlage für die Naturwissenschaften. Ich finde es faszinierend, wie man mit Mathematik in der Lage ist, komplexe Vorgänge zu beschreiben und

\section{Wolfram Koepf}

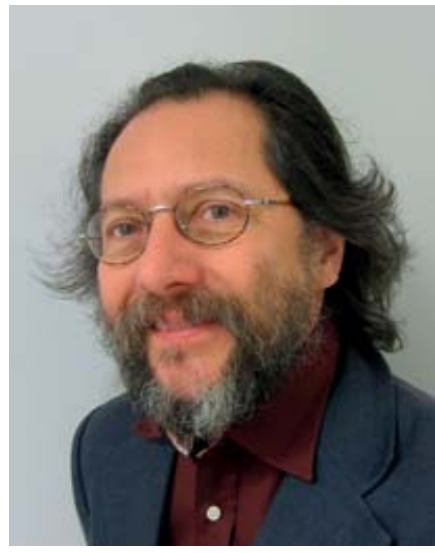

Geboren am 16. Juli 1953 in Stuttgart. 1972 Abitur. Studium der Mathematik und Physik an der Universität Stuttgart und an der Freien Universität Berlin. 1981 Diplom, 1984 Promotion und 1990 Habilitation in Mathematik an der FU Berlin mit Arbeiten in der Geometrischen Funktionentheorie. wie man helfen kann, Wirkungszusammenhänge zu erkennen.

In der Lehre versuche ich, Studierenden von Anfang an Zusammenhänge zwischen dem Stoff der Vorlesung und weiterführenden Veranstaltungen oder unserer Umgebung (Form der Bienenwaben, Dach des Münchener Olympiastadions, Computertomograph, Optionsbewertung, Kreditkartenverschlüsselung etc.) zu vermitteln. Ebenso versuche ich, die Vorlesung in den Kontext der Geschichte einzuordnen. Studierende, insbesondere auch des Mathematik-Lehramts, sind häufig überrascht, wie viel Energie ich in eine Vorlesung stecken kann. Sie merken schnell, dass ich sie ernst nehme und auf ihre spezielle Situation eingehe, dafür dann allerdings auch Mitarbeit und Leistung einfordere. In den Jahren 2012 und 2013 war ich Fachgutachter des Landes NRW für Abituraufgaben.

Wie im kurzen Lebenslauf oben ersichtlich wird, engagiere ich mich stark in der Selbstverwaltung meiner Universität. Ich denke, dass Mathematikerinnen und Mathematiker Gremienarbeit häufig meiden, weil ihrer Meinung nach dort viel Zeit vergeudet wird. Dennoch sollten viele von uns an Universitäten und in der Öffentlichkeit aktiv mitsprechen, damit wir im Wettstreit um Ressourcen, vor allem aber auch bei den ständigen Diskussionen um Veränderungen des Bildungssystems, gehört werden. Ich möchte ebenso dazu beitragen, dass Mathematik in unserer Gesellschaft als positiv und wichtig wahrgenommen wird. Daher kandidiere ich gerne noch einmal für das Präsidium der DMV.

In der vergangenen Amtsperiode war ich zuständig für Kontakte der DMV zur Industrie. Zusammen mit den Partnern vor Ort habe ich auf den Jahrestagungen in Hamburg und Braunschweig das Mittagsseminar „Mathematik in Industrie und Gesellschaft" organisiert. Ich freue mich, dass diese Veranstaltung so gut angenommen wurde. Ich plane, diese Reihe fortzusetzen und die bestehenden Kontakte zu intensivieren.

Nach meiner Habilitation habe ich mein Forschungsgebiet gewechselt. Ich arbeite seitdem auf den Gebieten der Computeralgebra und der Speziellen Funktionen. Ermöglicht wurde dies unter anderem durch ein Stipendium der Alexander von Humboldt-Stiftung. Lehrbücher zum Einsatz von Computeralgebra in Schule und Lehre, eine Monografie über Summationsalgorithmen und weitere Lehrbücher über die Konstruktion der reellen Zahlen und über Computeralgebra runden meine Tätigkeiten ab.

Seit Januar 1996 bin ich Mitglied der Leitung der Fachgruppe Computeralgebra, und von 2002 bis 2011 war ich ihr Sprecher. 1997 bis 2000 war ich als Professor für angewandte Mathematik an der Hochschule für Technik, 
Wirtschaft und Kultur Leipzig beschäftigt und seit 2000 bin ich als Professor für Computeralgebra an der Universität Kassel tätig und dortiger DMV-Ansprechpartner.

Seit 2009 bin ich Mitglied des DMV-Präsidiums und als solches bislang für den Webauftritt mathematik.de zuständig. Ferner leite ich aktuell als Vertreter der DMV die Mathematik-Kommission Übergang Schule-Hochschule, die 2011 von den drei Fachgesellschaften DMV, GDM und MNU eingerichtet wurde und sich unter anderem für eine akteptable Umsetzung der Abiturstandards einsetzt. Die Kommission hat bereits drei Tagungen zum Übergangsthema organisiert, die letzte fand vom 29.-31. Mai 2017 in

\section{Andrea Peter-Koop}

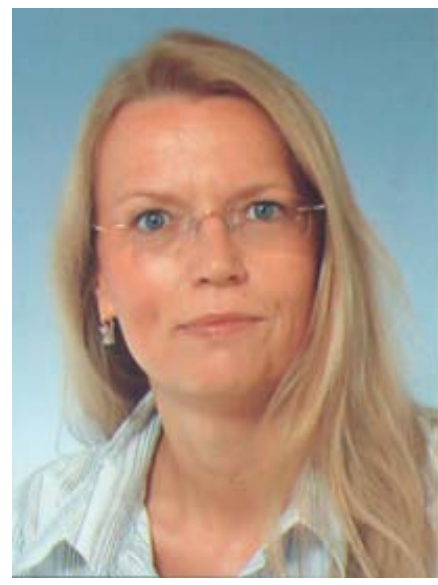

Geboren 1965 in Bielefeld, Studium des Lehramtes Primarstufe an der Universität Bielefeld mit den Fächern Mathematik, Deutsch, Sachunterricht, 1990 Erstes Staatsexamen und im Anschluss zweijähriger Forschungsaufenthalt in Melbourne, Australien, Anfang 1995 Promotion zum Dr. phil. bei Prof. Hans-Georg Steiner in Bielefeld zum

Thema Lehrerfortbildung aus international vergleichender Perspektive. Direkt im Anschluss bis 2001 Wissenschaftliche Assistentin am Lehrstuhl von Prof. Peter Sorger in Münster und nach Lehrstuhlvertretungen in Gießen, Heidelberg und Oldenburg 2003 Ruf auf eine Professur für Didaktik der Mathematik an der Universität Oldenburg, seit 2011 Professorin am IDM an der Universität Bielefeld. Seit der Berufung als Professorin war ich mehrfach als Gastprofessorin im Ausland, unter anderem an der University of Port Elizabeth in Südafrika und der Zhejiang International Studies University in Hangzhou, China.

In Bezug auf meine Forschung und Lehre sowie auch im Rahmen meines ehrenamtlichen Engagements liegt mir die Förderung mathematisch besonders leistungsstarker und interessierter Schülerinnen und Schüler ebenso am Herzen wie die Prävention und Therapie von Rechenschwierigkeiten. So habe ich während meiner Münsteraner Zeit damit begonnen, spezielle Angebote für mathematisch besonders interessierte Grundschulkinder zu machen und diese Aktivitäten mit der Lehrerausbildung zu verknüpfen. In Niedersachsen war ich von 2006 bis 2008 zudem Jury-Mitglied beim Landeswettbewerb Jugend forscht - Kinder experimentieren für den Bereich Mathematik/Informatik. Für die DMV bin ich seit Beginn des Jahres im Wissenschaftlichen Beirat von Mathe im Advent
Münster statt und hatte das hochaktuelle Thema Mathematik in Schule und Hochschule - wie groß ist die Lücke und wie gehen wir mit ihr um? Hier haben wir versucht, die oft sehr unterschiedlichen Meinungen zusammenzuführen.

Ich habe mich dazu bereit erklärt, der Kommission in den nächsten Jahren weiterhin zur Verfügung zu stehen, sofern ich dem DMV-Präsidium angehöre. Ferner möchte ich gerne nach Umstellung der DMV-Webseiten auch in Zukunft dafür sorgen, dass unsere Rezensionsdatenbank wächst und Rezensionen neuer Bücher aufgenommen werden. Daher bitte ich um Ihre Stimme.

und beteilige mich aktiv an der aufzubauenden Begleitforschung.

Bereits seit meiner Promotion verfolge ich zudem das Thema Lehrerbildung auf unterschiedlichen Ebenen, seit 2001 als Herausgeberin der Buchreihe Mathematics Teacher Education and Development bei Springer sowie als Koordinatorin diesbezüglicher Topic Groups auf internationalen Tagungen, als Wissenschaftlerin im Rahmen eigener Forschungsprojekte, aktuell in einem interdisziplinären Drittmittelprojekt zur Entwicklung (mathematik-) diagnostischer Kompetenzen im Studium sowie in einem internationalen Drittmittel-Forschungsprojekt Learning from Lessons, gefördert durch den Australian Research Council, gemeinsam mit australischen und chinesischen Partneruniversitäten. Dieses Projekt geht der Frage nach, wie Mathematiklehrer/-innen durch ihr Unterrichtshandeln lernen und wie dieses Lernen optimiert werden kann.

Darüber hinaus beschäftigt mich das Thema Lehrerbildung auch hochschulpolitisch als stellvertretende Direktorin der Bielefeld School of Education. Aus dieser Perspektive verfolge ich auch seit einigen Jahren mit großem Interesse die Beteiligung der DMV an Diskussionen und Papieren zur Lehrerausbildung. Daher freue ich mich sehr über den Vorschlag, erneut für das Präsidium zu kandidieren, um mich dort für die Aus-, Fort- und Weiterbildung von Mathematiklehrer/-innen einzusetzen, nicht nur aber auch angesichts der aktuellen Herausforderungen im Kontext von Inklusion und einer erheblichen Zahl geflüchteter Kinder und Jugendlicher an unseren Schulen. 